\title{
Aiweixin, a traditional Uyghur medicinal formula, protects against chromium toxicity in caenorhabditis elegans
}

Binggen Zhu ${ }^{1,2^{*}}$, Ping Yang ${ }^{3}$, Nurahmat Mammat ${ }^{1}$, Hui Ding ${ }^{4}$, Junmin He ${ }^{1,5}$, Yong Qian ${ }^{4}$, Jian Fei ${ }^{3,5}$ and Kaiser Abdukerim ${ }^{1 *}$

\begin{abstract}
Background: Aiweixin (AWX) is a traditional Uyghur medicine prescription, and has been mainly used to treat heart and brain diseases for a long time. Previous studies indicated that AWX had therapeutic effects in a rat model of myocardial ischemia reperfusion injury. In this study, we investigate whether AWX has protective effects against chromium toxicity in Caenorhabditis elegans (C. elegans).

Methods: The AWX decoction was the conventional product for clinical use. It was added into M9 buffer in a certain volume for the treatment to the wild-type C. elegans and mutational worms, daf-16, glp-1 (notch), daf-2, rsks-1 and eat-2. Assays for hexavalent chromium $\{\mathrm{Cr}(\mathrm{VI})\}$ stress and reactive oxygen species (ROS) production were used.

Results: We found that AWX at moderate contents $(0.083,0.1,0.125$ volume of AWX/total volume) increased resistance of $C$. elegans to $C r(\mathrm{VI})$ exposure, although higher contents of $A W X$ are toxic for $C$. elegans. The protective effect of AWX was DAF-16-dependent, but independent on the DAF-2, GLP-1, RSKS-1 and EAT-2. AWX (0.1 volume of AWX/total volume) significantly reduced ROS production of $C$. elegans induced by $\mathrm{Cr}(\mathrm{VI})$ exposure.
\end{abstract}

Conclusion: These results indicated the AWX protected against the toxicity of $\mathrm{Cr}(\mathrm{VI})$ in $\mathrm{C}$. elegans, and the oxidative stress protective mechanism in worms should be involved.

Keywords: Aiweixin, Traditional Uyghur Medicine, Chromium Toxicity, Caenorhabditis elegans

\section{Background}

Aiweixin (AWX) is a traditional Uyghur medicine prescription and consists of 15 ingredients including Dracocephalum moldavicum L., Eletteria cardamomum (L.) Maton, Salix caprea L. (Salicaceae) flowers, Lavandula augustifolia (lavender), Borago officinalis L. (Boraginaceae) stems and leaves, Borago officinalis L. (Boraginaceae) flower, Nardostachys jatamansi DC. root and rhizome (Nardostachyos Radix et Rhizoma), Bombyx mori (Abresham) silk cocoons, Usnea longissima Ach., Rosa rugosa Thunb. flowers, Syzygium aromaticum L., Lindera caudata (Nees) Hook.f., Myristica fragrans (Houtt.), Crocus sativus L. and Moschus [1]. AWX is orally administered as a decoction. According to the theory of traditional

\footnotetext{
*Correspondence: bgenz2003@aliyun.com; kaiserrena86@aliyun.com 'College of Xinjiang Uyghur Medicine, Hetian, Xinjiang 848000, China ${ }^{2}$ Tongji University School of Medicine, Shanghai 200092, China Full list of author information is available at the end of the article
}

Uyghur medicine, AWX has preventive and treatment effects in many aspects, including modulating "Mizaj", balancing body fluid, improving blood circulation, strengthening the function of heart and brain, etc. It has been used to treat diseases induced by abnormal "savda", such as coronary heart disease, myocardial ischemia, arrhythmia, cerebral infarction, depression [1, 2]. The effectiveness of AWX has been well documented during long-term clinical practice. Previous pharmacological experiments demonstrated that AWX exerted therapeutic effects in a rat model of myocardial ischemia reperfusion injury, possibly via alleviation of oxidative stress $[3,4]$.

Chromium exists mostly in two valence states in nature: hexavalent chromium $\{\mathrm{Cr} .(\mathrm{VI})\}$ and trivalent chromium [5]. The trivalent chromium is essential for organisms, and is acknowledged as a dietary supplement. But the $\mathrm{Cr}(\mathrm{VI})$ and its compounds have been recognized as having potential severe adverse effects on health. 
Previous studies demonstrated that $\mathrm{Cr}(\mathrm{VI})$ induces oxidative stress, DNA damage, apoptotic cell death and altered gene expression [5-7]. The Caenorhabditis elegans (C. elegans), a free living nematode that lives mainly in the liquid phase of soils, is considered as an ideal model organism because of its short life span, ease of manipulation, and low cost. It has been found favor as a valuable bioindicator organism in metal toxicity study for its best-characterized properties at the genetic, physiological, molecular, and developmental levels $[8,9]$. Assays using C. elegans to observe the lethality and sublethal endpoints, growth, reproduction, lifespan, locomotion behavior, stress response, and oxidative damage have been well developed to monitor the toxicity of heavy metals including $\mathrm{Cr}(\mathrm{VI})$ [8]. In the present study, we investigate whether AWX has protective effects against $\mathrm{Cr}(\mathrm{VI})$ toxicity in $C$. elegans, in order to get more preclinical data which support the therapeutic effects of AWX.

\section{Methods}

\section{Preparation and phytochemical profile of AWX}

The AWX decoction was the conventional product for clinical use, manufactured by Xinwei Pharmaceutical Factory (Hetian, Xinjiang Uyghur Autonomous Region, P. R. China). According to the Pharmacopeia of P. R. China [1], the product is made from Dracocephalum moldavicum L. (15 g), Eletteria cardamomum (L.) Maton (15 g), Salix caprea L. (Salicaceae) flowers (10 g), Lavandula augustifolia (lavender) (15 g), Borago officinalis L. (Boraginaceae) stems and leaves (10 g), Borago officinalis L. (Boraginaceae) flower (10 g), Nardostachys jatamansi DC. root and rhizome (Nardostachyos Radix et Rhizoma) (10 g), Bombyx mori (Abresham) silk cocoons (50 g), Usnea longissima Ach. (3 g), Rosa rugosa Thunb. flowers (15 g), Syzygium aromaticum L. (15 g), Lindera caudata (Nees) Hook.f. (10 g), Myristica fragrans (Houtt.) (15 g), Crocus sativus L. $(0.6 \mathrm{~g})$ and Moschus $(0.2 \mathrm{~g})$. The AWX decoction was prepared as follows: before 13 dry herbs were smashed into rude powder, decocted and boiled 3 times (1.5 h for each) while $300 \mathrm{ml}$ of distilled water was collected. The decocted liquid merged the $300 \mathrm{ml}$ of distilled water was filtrated, concentrated, and added with ethanol to $70 \%$ the content of ethanol. The decoction was filtrated again after $24 \mathrm{~h}$ while ethanol was collected. Meanwhile, after the two dry medicinal materials was put in $70 \%$ ethanol solution, were extracted by reflux two times $(2 \mathrm{~h}$ for each). The extracted solution was filtrated while ethanol was collected. The former decoction and the latter extracted solution were mixed and added with distilled water to $1000 \mathrm{ml}$, then filtrated and sterilized.

For the quality control of AWX decoction, highperformance liquid chromatography-diode array detection
(HPLC-DAD) method has been used, referred to the previous paper [10]. The chromatographic separation was performed by a UItimate XB-C18 $(250 \mathrm{~mm} \times 4.6 \mathrm{~mm}$, $5 \mu \mathrm{m})$ column in Agilent 1200 (Agilent Technologies). Mobile phase was a gradient of Methanol-acetic acid water gradient. The effluent was monitored on a DAD detector. The fingerprint was set up on the data at $280 \mathrm{~nm}$. The Fig. 1 showed the HPLC fingerprint of three separated AWX samples. By using HPLC-UV-ESI-TOFMS system, 4 peaks were recognized according to compound molecular weight data. The highest peak 1 contains chebulic acid, while peak 2-4 represent gallic acid, protocatechuic acid, and eugenol respectively. However, others (more than 10 low peaks) were unidentified.

\section{Caenorhabditis elegans strain and maintenance}

The wild-type C. elegans strain N2 (Bristol) and mutational worms, daf-16 (mu86), glp-1(e2141), daf-2 (e1370), rsks-1 (ok1255) and eat-2 (ad465) were provided by the Caenorhabditis Genetics Center (University of Minnesota, Minneapolis, MN). Nematodes were generally incubated at $20^{\circ} \mathrm{C}$ on nematode growth media (NGM) plates with $\mathrm{E}$. coli OP50. For culture of daf-2 (e1370), and its wild-type control, nematodes were firstly developed at $16{ }^{\circ} \mathrm{C}$ for 3 days and then transferred to $21^{\circ} \mathrm{C}$ for the desired stage of development.

\section{Chromium stress assay and AWX treatment to C. elegans}

The procedure of chromium stress assay was based on our previous studies [11]. Synchronized Day 3 adult worms were collected and washed with M9 buffer for three times to remove the OP50 bacteria. Approximately 35 adults were suspended into each well of a 48-well culture plate containing $120 \mu \mathrm{l}$ M9 buffer with or without certain content of AWX $(0.05,0.067,0.083,0.1$, 0.125 volume of $\mathrm{AWX} /$ total volume). $30 \mathrm{~min}$ later, K2Cr2O7 $\{\mathrm{Cr}(\mathrm{VI})$, Sangon Biotech, Shanghai, China\} was added at final concentration of $10 \mathrm{mM}$, except for the experiments without $\mathrm{Cr}(\mathrm{VI})$ exposure. The plates were cultivated at $20{ }^{\circ} \mathrm{C}$ for certain time. Survived nematodes were counted at different time points. The nematodes were judged to be dead if they did not respond to stimulus using a small, metal wire. Each experiment was reiterated for at least three times.

\section{Reactive oxygen species (ROS) production}

The method of worms received $\mathrm{Cr}(\mathrm{VI})$ exposure and treatment of AWX was same as above described in the chromium stress assay. The assay of ROS production was based on published studies [7]. The examined nematodes were transferred to $0.5 \mathrm{~mL}$ of $\mathrm{M} 9$ buffer containing $5 \mu \mathrm{M}$ CM-H2DCFDA (Sigma-Aldrich, USA) and pre-incubated for $3 \mathrm{~h}$ at $20{ }^{\circ} \mathrm{C}$, and then mounted on $2 \%$ agar pads and examined with a fluorescence 


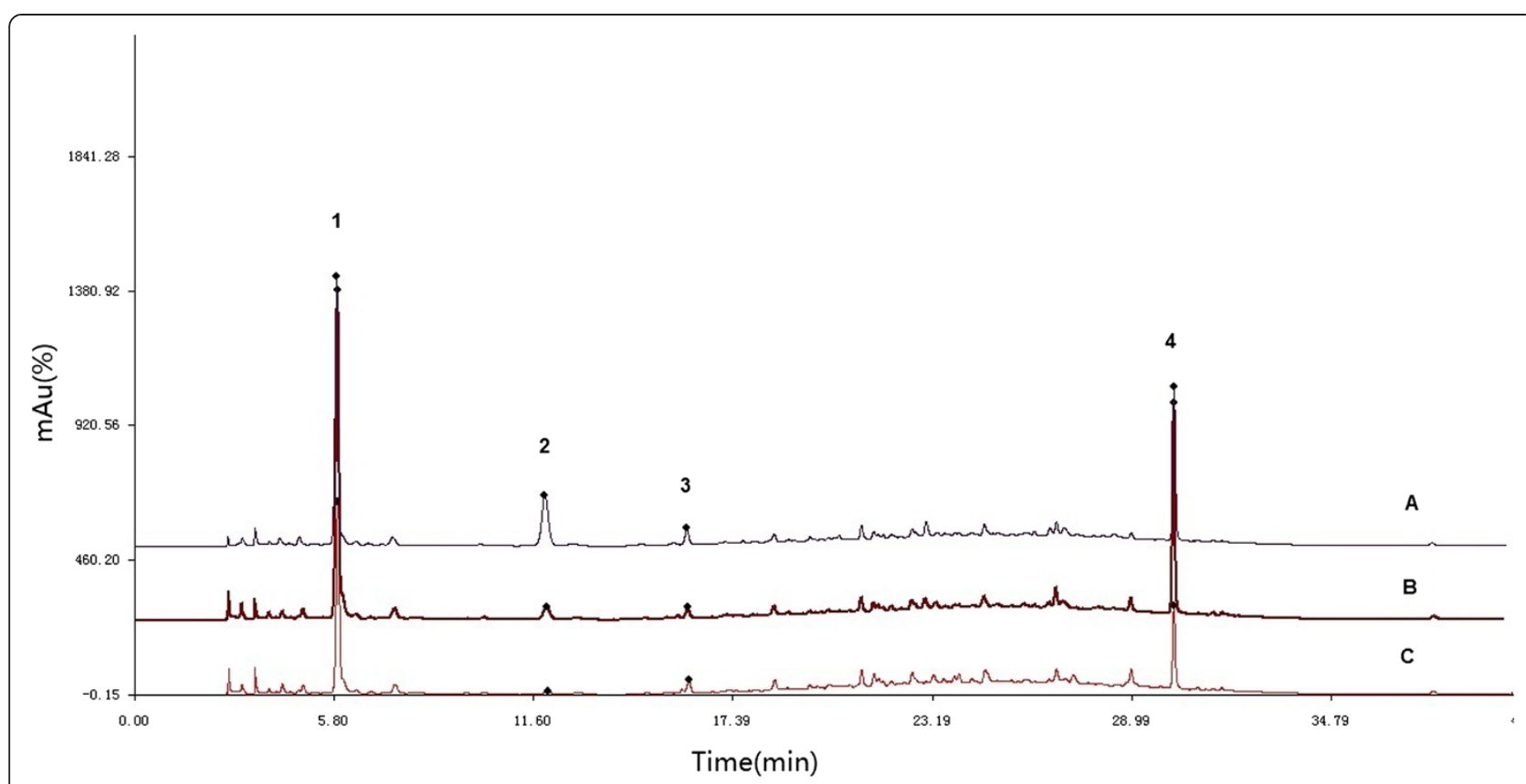

Fig. 1 The HPLC fingerprint of three separated AWX samples. A, B and C represented three separated AWX samples

microscope (Nikon, SMZ 1500, Japan) at 480/40 nm of excitation wavelength and 535/50 $\mathrm{nm}$ of emission filter. Fluorescence levels were measured using Image Software (NIS-Elements D3.1) by determining average pixel intensity in each animal. More than 30 animals were counted for the statistical. The relative fluorescence intensities of the worm were semiquantified. The semi-quantified ROS was expressed as relative fluorescent units (RFU). Three replicates were performed.

\section{Statistic}

The results are reported as mean \pm SEM. Significant differences between groups were tested by one-way analysis of variance (ANOVA) followed by Student's $t$ test. Differences among multiple means were assessed by one-way ANOVA with Bonferroni correction. Statistical software OriginPro 7.5 was used (http:// www.originlab.com/). Probability values less than or equal to 0.05 were considered statistically significant.

\section{Results}

High contents of AWX are toxic for C. elegans

All of synchronized day 3 adult worms used in our experiments survived well after $16 \mathrm{~h}$ incubation in M9 buffer without any additional compositions (seen the Fig. 2). Most (about $91.0 \%$ ) of worms were still in good condition after $23 \mathrm{~h}$, but the overwhelming majority was not alive at $38 \mathrm{~h}$ incubation. To understand the basic effects of AWX for C. elegans, the toxicity and safety of AWX were assessed. As shown in Table 1, the mortality of C. elegans was increased with the elevation of AWX contents, after $16 \mathrm{~h}$ incubation. AWX at 0.1 volume of AWX/total volume was a little of toxicity (death ratio was $3 / 34,8.8 \%$ ), but AWX at 0.2 and 0.333 were very toxic \{death ratio was $37 / 46(80.4 \%)$ and $45 / 45$ (100\%), respectively\}. The worms incubated with AWX at 0.067 and 0.083 lived as well as the ones without the treatment of AWX.

AWX increased resistance of $C$. elegans to $\mathrm{Cr}(\mathrm{VI})$ exposure in a time and dose-dependent manner

To investigate the protective effect of AWX against heavy metal $\mathrm{Cr}(\mathrm{VI})$ toxicity in $\mathrm{C}$. elegans, chromium stress assay was used. As shown in Fig. 3, the survival fraction of the N2 adults' worm received AWX at o.1 was significantly higher than the control without

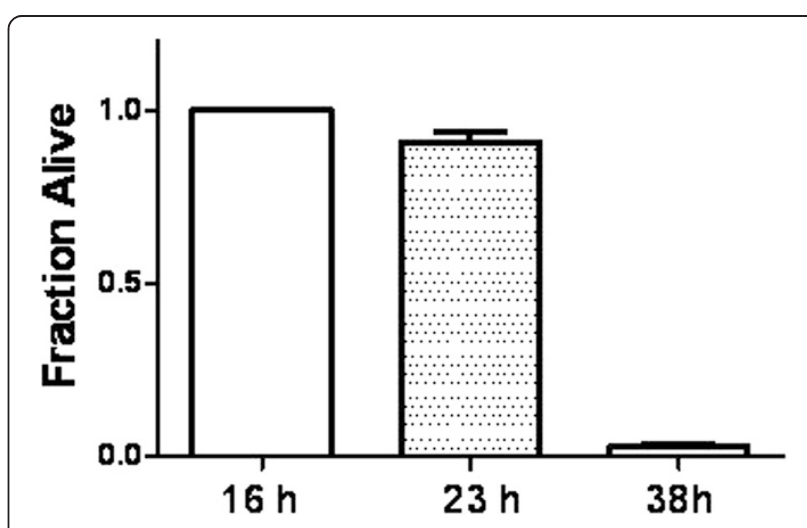

Fig. 2 The survival measurement of wild-type C. elegans which did not receive any treatments 
Table 1 Toxicity and safety of AWX for C. elegans

\begin{tabular}{lllllll}
\hline & \multicolumn{5}{l}{ Contents of AWX (volume of AWX / total volume) } \\
\cline { 2 - 7 } & 0 (control) & 0.067 & 0.083 & 0.1 & 0.2 & 0.333 \\
\hline Survival-16 h & 38 & 49 & 36 & 31 & 9 & 0 \\
Death-16 h & 0 & 0 & 0 & 3 & 37 & 45 \\
Total & 38 & 49 & 36 & 34 & 46 & 45 \\
\hline
\end{tabular}

treatment of AWX after $18 \mathrm{~h}(\mathrm{p}<0.01), 22.5 \mathrm{~h}(\mathrm{p}<0.01)$ and $24 \mathrm{~h}(\mathrm{p}<0.001)$ incubation. The similar effects of AWX at 0.083 and 0.125 were also seen, but not as strong as AWX at 0.1. The effects of AWX at 0.05 and 0.067 were not significant compared to the control. The significant enhanced effect at $24 \mathrm{~h}$ disappeared in the groups of AWX at 0.083 and 0.125 , and was only observed in the group of AWX at 0.1. Moreover, according to the data in Fig. 2, most (about $91.0 \%$ ) of worms without any treatments were alive at $23 \mathrm{~h}$, but about only $39.2 \%$ of worms in the control to be exposure to $\mathrm{Cr}(\mathrm{VI})$ survived at near this time point $(22.5 \mathrm{~h})$ (seen the Fig. 3 ). In contrast, AWX at 0.1 significantly elevated the fraction of survival worms roughly to $75.6 \%$.

\section{The protective effect of AWX was DAF-16-dependent}

For further researches on the action of AWX, several of characterized mutant nematode lines were used. As Fig. 4 showed, AWX at 0.1 had significant protective effects on the wild-type worms after $16 \mathrm{~h}(\mathrm{p}<0.05), 18 \mathrm{~h}(\mathrm{p}<0.01)$, $20 \mathrm{~h}(\mathrm{p}<0.001)$ and $21 \mathrm{~h}(\mathrm{p}<0.001)$ incubation, but no significant influence on the mutational worm, daf-16 (mu86), implying that the DAF-16 function was essential for the action of AWX. However, experiments with other related mutant lines of worms, including $g l p-1(e 2141)$,

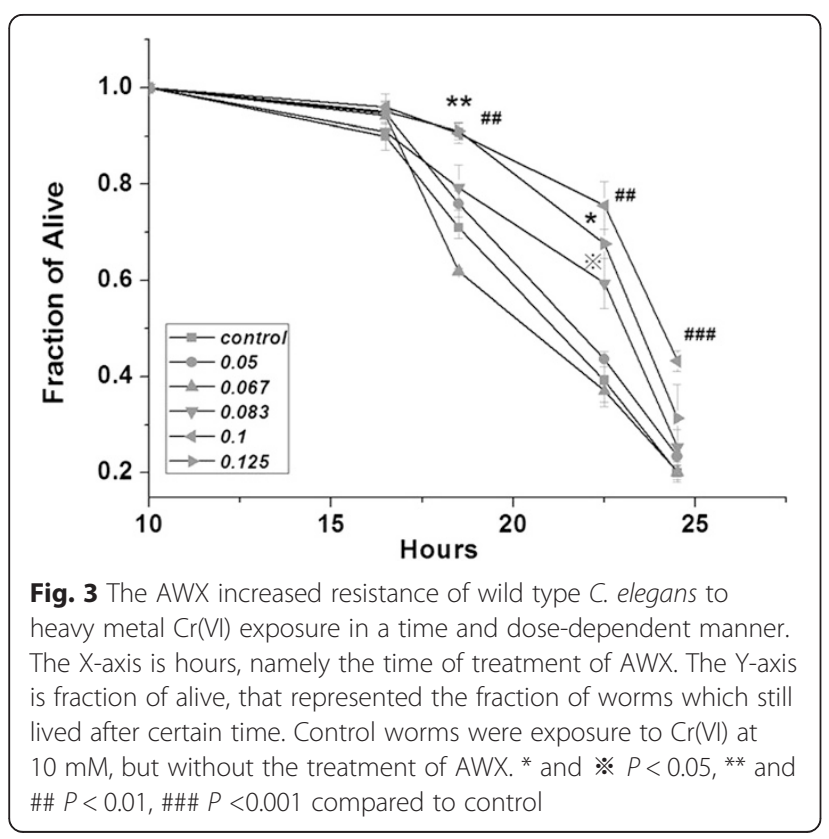

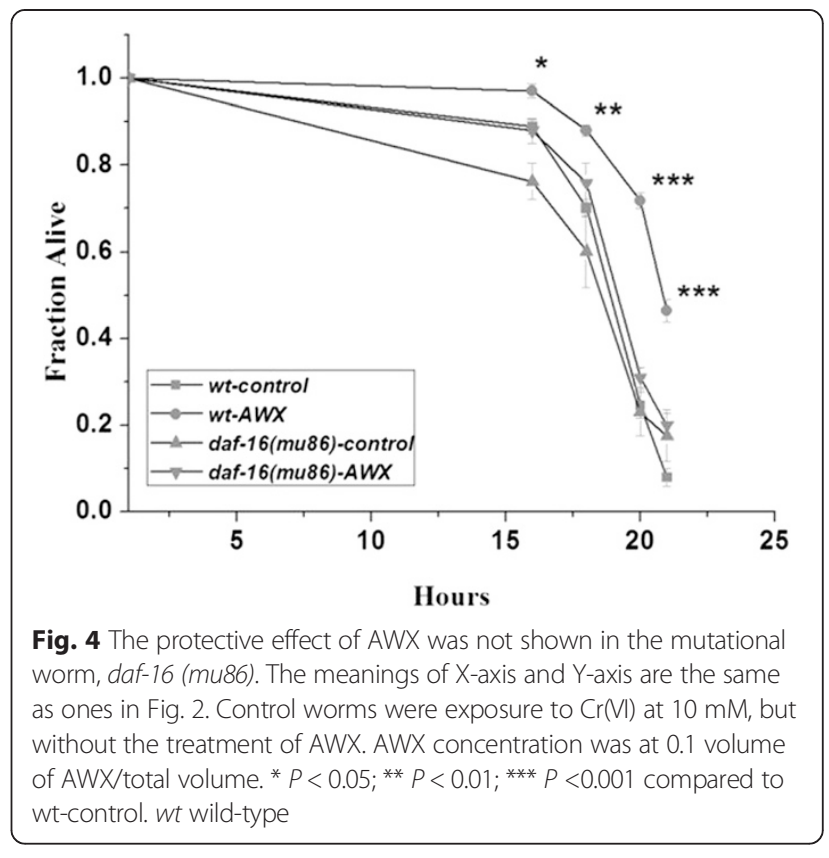

daf-2(e1370), rsks-1(ok1255) and eat-2(ad465), demonstrated protective effects of AWX as same as in the wild-type worms (seen in Fig. 5), indicating that the protective effect of AWX was independent on the function of GLP-1, DAF-2, RSKS-1, and EAT-2.

\section{AWX reduced ROS production of $C$. elegans induced by $\mathrm{Cr}(\mathrm{VI})$ exposure in a time-dependent manner}

Chromium exposure increased the ROS production of nematodes [7]. To quantify whether AWX treatment decreases ROS levels elevated by $\mathrm{Cr}(\mathrm{VI})$ exposure in C. elegans, the ROS production was assayed.As shown in Fig. 6, treatment of AWX at 0.1 significantly compromised the density of fluorescent or the elevation of ROS production after $16 \mathrm{~h}$ and $19.5 \mathrm{~h}(\mathrm{p}<0.05$ and $p<0.0005$, respectively). Although the decreased effect was not significant at $14 \mathrm{~h}(p<0.08)$, the effect of AWX appeared to begin early, and became stronger with the extension of incubation time, to some degree.

\section{Discussion}

The toxicity of $\mathrm{Cr}(\mathrm{VI})$ for $C$. elegans is well documented [7-9, 12, 13]. AWX (a traditional Uyghur medicine prescription), for the first time, was studied for the treatment of $\mathrm{Cr}(\mathrm{VI})$ intoxication in C. elegans. We, using endpoints of lethality, found that AWX at moderate contents $(0.083,0.1,0.125$ volume of AWX/total volume) had protective effects against the toxicity of $\mathrm{Cr}(\mathrm{VI})$ in C. elegans, although AWX at higher concentrations (more than or equal to 0.1 volume of $\mathrm{AWX} /$ total volume) was toxic. 

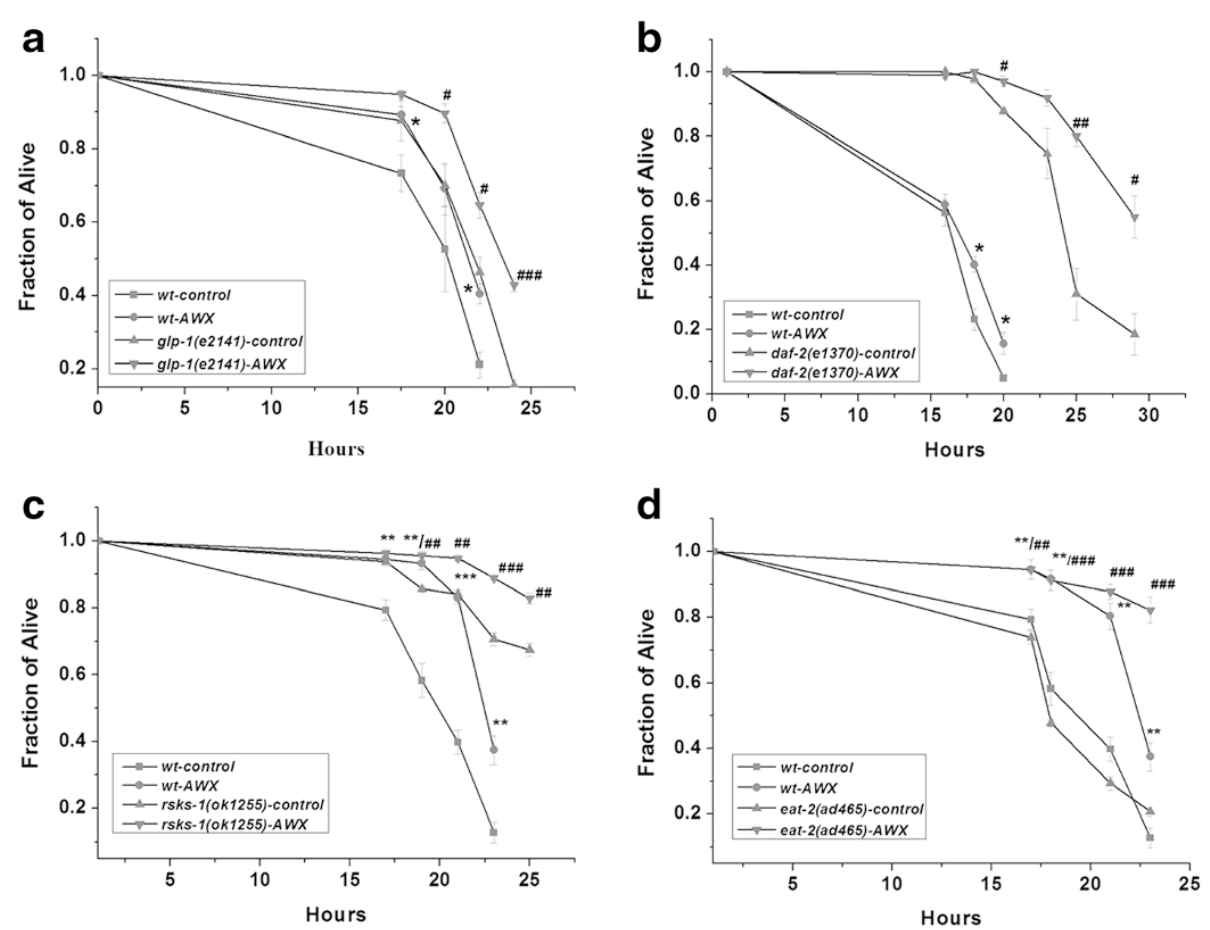

Fig. 5 The protective effect of AWX was shown in the mutational worms, glp-1(e2141) (a), daf-2(e1370) (b), rsks-1(ok1255) (c) and eat-2(ad465) (d). The meanings of $X$-axis and $Y$-axis are the same as ones in Fig.2. Control worms were exposure to $\mathrm{Cr}(\mathrm{VI})$ at $10 \mathrm{mM}$, but without the treatment of AWX. AWX concentration was at 0.1 volume of AWX/total volume. ${ }^{*} P<0.05$, ${ }^{* *} P<0.01$, ${ }^{* * *} P<0.001$ compared to wt-control; \# $P<0.05$, \#\# $P<0.01$, \#\#\# $\mathrm{P}<0.001$ compared to mutant-control respectively. wt, wild-type

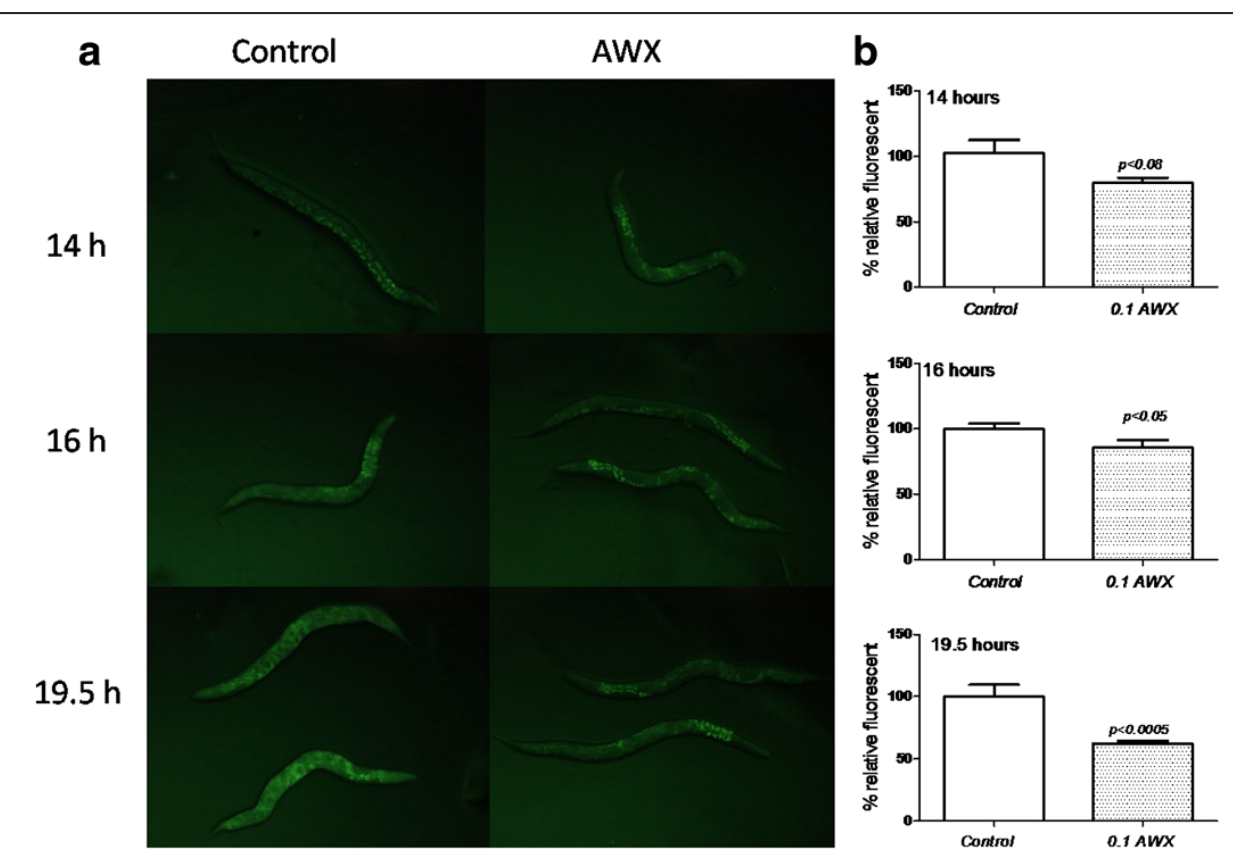

Fig. 6 ROS production of nematodes exposed to $\mathrm{Cr}(\mathrm{VI})$. (a) Pictures showing the ROS production as detected by CM-H2DCFDA labeling in nematodes exposed to $\mathrm{Cr}(\mathrm{VI})$ with or without AWX treatment. (b) Quantitative comparison of ROS production in nematodes exposed to $\mathrm{Cr}$ (VI) with or without AWX treatment. Control worms were exposure to $\mathrm{Cr}(\mathrm{VI})$ at $10 \mathrm{mM}$, but without $\mathrm{AWX}$ treatment. AWX concentration was at 0.1 volume of AWX/total volume 
The mechanistic cytotoxicity of $\mathrm{Cr}(\mathrm{VI})$ is not completely understand, however, a large number of studies demonstrated that $\mathrm{Cr}(\mathrm{VI})$ induces oxidative stress, DNA damage, apoptotic cell death and altered gene expression [5]. Concentration and time-dependent effects of $\mathrm{Cr}(\mathrm{VI})$ were demonstrated on increased ROS production and subsequent lipid peroxidation, enhanced excretion of urinary lipid metabolites, DNA fragmentation and apoptotic cell death in both in vitro and in vivo models [5]. According to our preliminary analysis regarding to the components of AWX, it contains chebulic acid, gallic acid, protocatechuic acid, and eugenol. Without exception, all of these compounds are potent antioxidants, by reviewing the literatures [14-18]. Moreover, considering that the AWX showed anti-oxidative effects in the previous studies in rats $[3,4]$, we supposed that AWX might protect the $C$. elegans from the oxidative damage induced by the $\mathrm{Cr}(\mathrm{VI})$. The experiment of ROS production demonstrated that AWX significantly reduced ROS production of $C$. elegans induced by $\mathrm{Cr}(\mathrm{VI})$ exposure. This result supports our hypothesis. The further indirect evidence comes from the studies used several mutant nematode strains.

The effect of AWX was dependent on the DAF-16 function. The DAF-16 is a forkhead transcription factor, which integrates signals from multiple pathways and regulates its downstream target genes to control diverse processes $[19,20]$. It is an important signal transducer of the insulin/IGF-1 signaling pathway, and it also receives input from several other pathways that regulate life span and the germline $[19,20]$. Our further experiments demonstrated that the protective effect of AWX was independent on the functions of DAF-2 (insulin-IGF receptor), GLP-1(notch, regulating self-renewal and differentiation of germ stem cells), RSKS-1 (worm homolog of mammalian p70S6K, promoting cell cycle progression in the germ line) and EAT-2 (involving in life span control), implying that the insulin/IGF-1 pathway and pathways regulating life span (through diet restriction) and germline were not involved [21-24]. Therefore, the further pathway of AWX affecting on DAF-16 is not clear yet, and awaits further studies. While DAF-16 translocates into the nucleus, it binds dozens of target promoters of genes directly, and acts as an activator or a repressor of transcription. These genes participate in stress protection, the promotion or prevention of longevity, dauer formation/maintenance and fat storage $[19,20]$. DAF-16 activates stress-response genes. The microarray and/or the bioinformatics studies disclosed that antioxidant genes (such as superoxide dismutase, metallothioneine, catalase, and glutathione $S$-transferase), and small heat shock protein genes are involved in the protective effects in worms $[19,20]$. Therefore, the intensive researches of these downstream target genes of DAF-16 should be helpful to elucidate the molecular mechanisms of AWX.

\section{Conclusions}

In conclusion, AWX, a traditional Uyghur medicine prescription, had protective effects against the toxicity of $\mathrm{Cr}(\mathrm{VI})$ in C. elegans. As AWX suppressed ROS production of $C$. elegans induced by $\mathrm{Cr}(\mathrm{VI})$ exposure, and the protection was dependent on the DAF-16 function, the oxidative stress protective mechanism in worms should be involved.

\section{Competing interests}

The authors declare that they have no competing interests.

\section{Authors' contributions}

BZ, NM, FJ and KA conceived and designed the study. PY, HD, JH, and YQ performed the experiments. BZ and NM wrote the manuscript. PY, HD, FJ and $Y Q$ analyzed data. BZ, NM and JH searched and reviewed literature. All authors read and approved the final manuscript.

\section{Acknowledgements}

The work was supported by the Science and Technology Support Project of Xinjiang Uyghur Autonomous Region, China (201233147).

\section{Author details}

${ }^{1}$ College of Xinjiang Uyghur Medicine, Hetian, Xinjiang 848000, China. ${ }^{2}$ Tongji University School of Medicine, Shanghai 200092, China. ${ }^{3}$ Shanghai Research Center for Model Organisms, Shanghai 201203, China. ${ }^{4}$ Shanghai Standard Biotech Co. Ltd, Shanghai 201203, China. ${ }^{5}$ School of Life Sciences and Technology, Tongji University, Shanghai 200092, China.

Received: 18 November 2014 Accepted: 16 July 2015

Published online: 18 August 2015

\section{References}

1. China Pharmacopoeia Commission. Drug Standard of Ministry of Public Health of the Peoples Republic of China (Uyghur Medicine). Urumgi: Xinjiang Publishing House of Science, Technology and Health; 1999. p. 72.

2. Julaiti T, Tuehong A, Wu Q. The clinical efficacy research of Aiweixin oral liquor in the treatment of coronary heart disease and myocardial ischemia. J Med Pharm Chin Minor. 2009;15:10-3.

3. Wang R, Wang L, Liu Y. Research on the protective effects of Aiweixin ischemia-reperfusion injury in liquid in myocardial rats. J Xinjiang Med Univ. 2009;32:146-9.

4. Yuan YM, Wang L, Wang R. Influence of Aiweixin oral liquor on MPO and MDA in MIRI rat. J Xinjiang Med Univ. 2010;33:33-5.

5. Bagchi D, Stohs SJ, Downs BW, Bagchi M, Preuss HG. Cytotoxicity and oxidative mechanisms of different forms of chromium. Toxicology. 2002;180:5-22

6. Bagchi D, Stohs SJ, Downs BW, Bagchi M, Preuss HG. Reply to the editor: Cytotoxicity and oxidative mechanisms of different forms of chromium. Toxicology. 2003;186:175-7.

7. Wu Q, Qu Y, Li X, Wang D. Chromium exhibits adverse effects at environmental relevant concentrations in chronic toxicity assay system of nematode Caenorhabditis elegans. Chemosphere. 2012;87:1281-7.

8. Leung MCK, Williams PL, Benedetto A, Au A, Helmcke KJ, Aschner M, et al. Caenorhabditis elegans: an emerging model in biomedical and environmental toxicology. Toxicol Sci. 2008;106:5-28.

9. Shen $\mathrm{L}$, Xiao J, Ye H, Wang D. Toxicity evaluation in nematode Caenorhabditis elegans after chronic metal exposure. Environ Toxicol Pharm. 2009;28:125-32.

10. Yang S, Zhou W, Zhang Y, Yan C, Zhao Y. Effects of Liuwei Dihuang decoction on ion channels and synaptic transmission in cultured hippocampal neuron of rat. J Ethnopharmacol. 2006;106:166-72.

11. Yang P, Sun R, Yao M, Chen W, Wang Z, Fei J. A C-terminal truncated mutation ofspr-3gene extends lifespan in Caenorhabditis elegans. Acta Biochim Biophys Sin. 2013:45:540-8.

12. Chen J, Jiang Y, Xu C, Yu L, Sun D, Xu L, et al. Comparison of two mathematical prediction models in assessing the toxicity of heavy metal mixtures to the feeding of the nematode Caenorhabditis elegans. Ecotoxicol Environ Saf. 2013;94:73-9. 
13. Xing $X$, Rui $Q$, Wan D. Lethality toxicities induced by metal exposure during development in nematode Caenorhabditis Elegans. Bull Environ Contam Toxicol. 2009;83:530-6.

14. Kakkar S, Bais S. A review on protocatechuic acid and its pharmacological potential. ISRN Pharmacol. 2014;952943:9. http://dx.doi.org/10.1155/2014/ 952943.

15. Korania MS, Farbood Y, Sarkaki A, Moghaddam HF, Mansouri MT. Protective effects of gallic acid against chronic cerebral hypoperfusion-induced cognitive deficit and brain oxidative damage in rats. Eur J Pharm. 2014;733:62-7.

16. Lee HS, Jung SH, Yun BS, Lee KW. Isolation of chebulic acid from Terminalia chebula Retz. and its antioxidant effect in isolated rat hepatocytes. Arch Toxicol. 2007:81:211-8.

17. Salcedo CL, Frías MA, Cutro AC, Nazareno MA, Disalvo EA. Antiradical activity of gallic acid included in lipid interphases. Biochim Biophys Acta. 1838;2014:2656-61.

18. Slamenová D, Horváthová E, Wsólová L, Sramková M, Navarová J. Investigation of anti-oxidative, cytotoxic, DNA-damaging and DNA-protective effects of plant volatiles eugenol and borneol in human-derived HepG2, Caco-2 and VH10 cell lines. Mutat Res. 2009;677:46-52.

19. Mukhopadhyay A, Oh SW, Tissenbaum HA. Worming pathways to and from DAF-16/FOXO. Exp Gerontol. 2006;41:928-34.

20. Murphy CT. The search for DAF-16/FOXO transcriptional targets: Approaches and discoveries. Exp Gerontol. 2006:41:910-21.

21. Baumeister R, Schaffitzel E, Hertweck M. Endocrine signaling in Caenorhabditis elegans controls stress response and longevity. J Endocrinol. 2006;190:191-202.

22. Byrd DT, Kimble J. Scratching the niche that controls Caenorhabditis elegans germline stem cells. Semin Cell Dev Biol. 2009;20:1107-13.

23. Korta DZ, Tuck S, Hubbard EJA. S6K links cell fate, cell cycle and nutrient response in C. elegans germline stem/progenitor cells. Development. 2012;139:859-70.

24. Lakowski B, Hekimi S. The genetics of caloric restriction in Caenorhabditis elegans. Proc Natl Acad Sci U S A. 1998;95:13091-6.

\section{Submit your next manuscript to BioMed Central and take full advantage of:}

- Convenient online submission

- Thorough peer review

- No space constraints or color figure charges

- Immediate publication on acceptance

- Inclusion in PubMed, CAS, Scopus and Google Scholar

- Research which is freely available for redistribution 